\title{
An Interactive Approach for Operation Evaluation: Temporal Planning as a Basis
}

\author{
Qiang Tao ${ }^{\text {a }}$, Tongzhou Zhao ${ }^{\text {b }}$, Dunbo Cai ${ }^{\text {c, * }}$ \\ Hubei Provincial Key Laboratory of Intelligent Robot, Wuhan Institute of Technology, Wuhan 430205, \\ China. \\ atao_qiang@163.com, bzhao_tongzhou@126.com, cdunbocai@163.com
}

\begin{abstract}
How to evaluate an operator (or its operations) reasonably is a very important and practical issue. However, many evaluation methods may work as a kind of post-hoc analysis and lack of interaction with the operator. In this paper, we proposed an interactive online evaluation approach that is based on automated temporal planning (TP). TP computes and keeps a set of the optimal plans. During the decision making process, the operator's operations are compared with those plans. Our approach can also predicate the long-term result of operator's operations, and provide appropriate suggestions. We developed a set of quantitative measures that are used to evaluate both the decisions made and the information gathering process. Applications of the approach were shown to be helpful for improving operator's decisions.
\end{abstract}

Keywords: Operation Evaluation; Operation Planning; Temporal Planning.

\section{Introduction}

Operations result from operators' decisions while they are facing events in some scenario. Usually, operators are firstly trained and then evaluated to get qualification. Evaluation of operations is very important in many areas of management [1-3], e.g., military operational plan. Unlike [1,2] and other domain-specific evaluation method, the work of [3] developed a general approach for evaluating operations. In terms of domain-specific, an evaluation method is designed with highly coupling with the knowledge or rules of the domain, i.e., it is often impossible to be adapted to other domains. In [3], Schubert et al proposed an evaluation methodology via the use of effects-based planning (EBP). EBP is a kind of goal directed, action-based, planning method. An action is modeled with preconditions on which it can execute, and effects which happen after the execution of the action. Specifically, the preconditions and effect are represented with logical propositions that may take values True or False. A representative system for EBP is the famous system known as STRIPS [5]. EBP can generate a sequence of actions (i.e., a plan) that when executed step by step, it can transfer the initial state into a (goal) state that satisfies some goal conditions. The principle of the work in [3] is to compare the operations made by human against the optimal plan(s) generated by an EBP planning system. Based on a set of sophisticated measures defined on the differences between the two plans, the operations of a human operator can be evaluated. As the problem description language PDDL [6] of EBP becomes expressive for a wide area of practical domains, the work of [3] can be applied on those domains generally.

However, the work of Schubert et al [3] only consider the problem settings where actions are instantaneous. Note that, in many practical working domains, actions take time; the delay of time has big impact on the expected result. To improve on the work of [3], in this paper we developed an approach to use temporal planning (TP) [6,7] for operation evaluation. In addition, we designed a set of reasonable measures for the analysis of human operations' decisions with respect to time. Temporal planning also provides a powerful tool to trace the change of resources, which is also exploited to guide an evaluation.

The rest of the paper is organized as follows: in the next section, we introduce the background of TP. Then, we present a TP-based evaluation method. After an example application of the proposed method, we make conclusions. 


\section{Background on Temporal Planning}

Temporal Planning is an extension of the classical STRIPS planning, where the execution of action takes time, and preconditions and effects is dependent on the action's time. For example, the action to fuel a tank, takes time in direct proportion to the free volume of it. Usually, when time is concerned, resource is also of importance. So, temporal planning is intrinsic to involve numeric resources. Formally, a task of TP is a tuple $\Pi=\left(V, A, I, G, T_{L}, \delta\right)$, where $V$ is the set of variables and $v \in V$ is associated with a domain $\operatorname{Dom}(v)$. A state $s$ is an assignment to the variables in $V$. $A$ is a set of actions. In the following, we use $[s, e]$ to represent an interval that starts at time point $s$ and end at $e$. An action $a \in A$ has the form $\left(d u r_{a}, C_{a}, E_{a}\right)$, where $d u r_{a} \in \mathfrak{R}$ is the duration, $C_{a}$ is the set of conditions, and $E_{a}$ is the set of effects. Each condition $c \in C_{a}$ has the form ([st $\left.t_{c}, e t_{c}\right] v \operatorname{cop}$ expr) where $s t_{c}$ and $e t_{c}$ are time points and $s t_{c} \leq e t_{c}$. The compare operator $\operatorname{cop} \in\{\geq,>, \leq,<,==\}$. The righthand expr is an expression composed by variables or values in the task. One effect $e$ of an action is of the form ([st $\left.t_{e}, e t_{e}\right] v$ op expr) and $o p \in\{+=,-=, *=,==,=\}$. Note that, for each action $a$, it have two variables $s_{a}$ and $e_{a}$ to hold the start and end times. Intuitively, $e_{a}=s_{a}+d u r_{a} . I$ is the initial state of the problem. $G$ is a set of conditions of form $(v=d)$, where $v \in V$ and $d \in \operatorname{Dom}(v) . T_{L}$ is the set of timed initial literals, and each $t i l \in T_{L}$ is of the form $\langle[t] f=d\rangle$ where $f \in V . \delta: A \rightarrow \Re$ is the cost function of actions. The planning problem on $\Pi$ is to find a sequence of actions $\left.\left.\pi=\left(\left\langle t\left(a_{1}\right), a_{1}\right)\right\rangle,\left\langle t\left(a_{2}\right), a_{2}\right), \ldots,\left\langle t\left(a_{m}\right), a_{m}\right)\right\rangle\right)$ when is executed on the initial state $I$, can reach a goal state that satisfies $G$.

Let's consider a simplified anti-missile task in military operations. When a missile named $m$ is found, the first operation pos_m_r is to determine its position with a radar $r$, and the second operation inter_m_im is to launch an intercept missile im. Suppose the positioning of $m$ with $r$ consumes energy 80, and takes 10 time units. The TP model of pos_m_r is as followings.

- $d u r_{p o s_{-} m_{-} r i}=10$

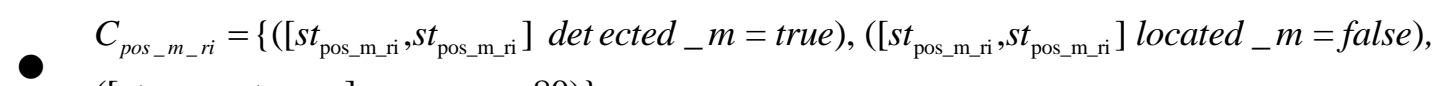

$\left(\left[s t_{\text {pos_m_ri }}, s t_{\text {pos_m_ri }}\right]\right.$ energy $\left.\left.>=80\right)\right\}$

- $E_{\text {pos_m_ri }}=\left\{\left(\left[e t_{\text {pos_m_ri }}, e t_{\text {pos_m_ri }}\right]\right.\right.$ located_m $=$ true $),\left(\left[s t_{\text {pos_m_i }}, s t_{\text {pos_m_ri }}\right]\right.$ enery $\left.\left.-=80\right)\right\}$

Note that, in the above model, the first condition $\left(\left[s t_{\text {pos_m_ri }}, s t_{\text {pos_m_ri }}\right]\right.$ detected_ $m=$ true) requires that at the start time of the action "the missile $m$ was detected already", the second

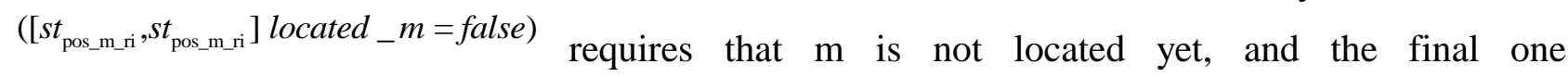
$\left(\left[s t_{\text {pos_m_i }}, s t_{\text {pos_m_ri }}\right]\right.$ energy $\left.>=80\right)$ requires the current level of energy to be greater than 80 .

\section{TP-based Operation Evaluation Method}

During the process of planning, human operators make decision dependent on a number of information or events, and following a set of operational rules. The will select a set of actions from sets of alternatives. We assume that a particular scenario that is used for training the operators is model by a TP task $\Pi=\left(V, A, I, G, T_{L}, \delta\right)$. Specifically, information is represented by variable value pairs. Events are described by timed initial literals $T_{L}$. The cost of each action is defined by $\delta$. The initial state and goal state of the scenario are defined by $I$ and $G$, respectively.

Suppose that the operational plan made by an human operator is $\pi^{h}=\left\langle\left(t\left(a_{0}^{h}\right), a_{0}^{h}\right\rangle,\left(t\left(a_{1}^{h}\right), a_{1}^{h}\right), \ldots,\left(t\left(a_{m}^{h}\right), a_{m}^{h}\right\rangle\right.$. The question is how to evaluate $\pi^{h}$, and what kinds of factors should be considered. We argue that the following 4 factors should be considered.

1) goal achievement The human operator is expected to select a plan to reach the goal of the task. Whether a goal is achieved or not and how many goals are not achieved are factors to evaluate.

2) information gathering Decisions are made on top a information. Some failed plan or unreasonable plan is caused by missing or neglecting some information. Therefore, the gap between the number of information gathered and the number that is required, is another factor. 
3) timeliness "Do the right thing at the right time" is a well known rules to management. Similarly, the action taken by the operator should on the right time. The offset to the desired start time should be considered.

4) costs To finish a task, different operators may use different plans. Although, the plans all are success, they may vary in cost. An experienced or calculating operator should select the plan with the lowest cost.

To obtain an optimal plan, we can use an optimal temporal planning system, such as the one developed in [8]. Suppose that the optimal plan is $\pi^{*}=\left\langle\left(t\left(a_{0}^{*}\right), a_{0}^{*}\right\rangle,\left(t\left(a_{1}^{*}\right), a_{1}^{*}\right), \ldots,\left(t\left(a_{m}^{*}\right), a_{m}^{*}\right\rangle\right.$. Next, we will evaluate $\pi^{h}$ with respect to $\pi^{*}$.

\subsection{Measurement of the goal achievement}

Following the semantics of executing an action on a state [6], we can simulate the execution of $\pi^{h}$ on the initial state $I$. Specifically, the execution of $a_{0}^{h}$ on $I$ results a state $s_{1}$ and the execution of $a_{1}^{h}$ on $s_{1}$ results the next state $s_{2}$. Generally, the execution of $a_{i}^{h}$ on $s_{i}$ results a state $s_{i+1}(i=0 . . m)$. Intuitively, $s_{m+1}$ is the final state resulted by $\pi^{h}$. The extent to which the goal is achieved is defined as:

$$
M_{G A}\left(\pi_{h}\right)=\frac{\left|\mathrm{s}_{\mathrm{m}+1} \cap G\right|}{|G|}
$$

Equation (1) first counts the number of goals achieved by the human operator's plan, and then divide it by the total number of goals required. The value of equation $(1)$ is in $[0,1.0]$.

\subsection{Measurement of the information gathering}

If the human operator's plan is different from the optimal plan, then we analyze how the information gathered is different from the expected. Let $a_{k}^{h}$ in $\pi^{h}$ is the last action that is the same as $a_{k}^{*}$ in $\pi^{*}$, then we define:

$$
M_{I G}\left(\pi_{h}\right)=\frac{\sum_{i=0}^{k}\left|C_{a_{i}^{b}}\right|}{\sum_{j=0}^{m}\left|C_{a_{j}^{k}}\right|}
$$

Note that, in equation (2), the denominator accounts for the total conditions (facts) needed to determine the optimal plan, and the numerator accounts for the facts collected by the human operator. The value of equation (2) is in $[0,1.0]$.

\subsection{Measurement of timeliness}

The action made by a human operator in each step should near the optimal time point. We compute the absolute offset of an action in $\pi^{h}$ relative to its counterpart in $\pi^{*}$. Then we sum up the offsets, and use the sum as an indicator of timeliness. Formally,

$$
M_{T L}\left(\pi_{h}\right)=\sum_{i=1}^{k}\left|s t\left(a_{i}^{h}\right)-s t\left(a_{i}^{*}\right)\right|
$$

In equation (3), the variable $k$ is the same as that in equation (2). Here, we use $s t(a)$ to represent the start time of an action $a$. If $k=m$, i.e., the operator's plan is logically reasonable, equation (3) reflects the accumulated offsets within the entire plan. We can see that the operator's plan $\pi^{h}$ with a higher $M_{T L}\left(\pi_{h}\right)$ is consider to be worse.

\subsection{Measurement of costs}

In a training scenario, we assume that each action $a$ is with cost $\delta(a)$. We prefer plans of lower cost. Usually, the cost of a plan is the sum of costs of its actions. To compare $\pi^{h}$ against $\pi^{h}$, in terms of cost, we define:

$$
\operatorname{cost}(\pi)=\sum_{a \in \pi} \delta(a)
$$

And

$$
M_{\text {cost }}\left(\pi_{h}\right)=\frac{\operatorname{cost}\left(\pi^{h}\right)}{\operatorname{cost}\left(\pi^{*}\right)}
$$

Note that the result of Equation (5) is in $[0, \infty]$. 


\section{Practical Application with Interactive Evaluation}

Based our defined measures in the previous section, we evaluate the current plan $\pi$ of a human operator with a measurement vector $M V(\pi):\left(M_{G A}(\pi), M_{I G}(\pi), M_{T L}(\pi), M_{\text {cost }}(\pi)\right)$. We use the $M V(\pi)$ in an interactive as is shown in Figure 1. In the work flow, we indicates the measurement vector of the operator's plan made so far, after each step of decisions. The operator could choose to continue its decision process or stop. This approach was deployed as a prototype tool for a military operational planning scenario, similar as the scenario described in [3].

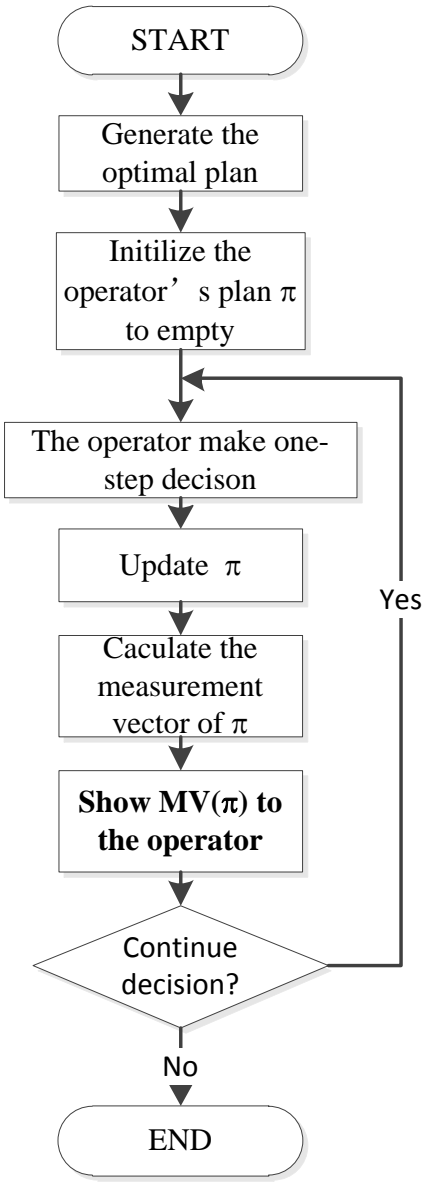

Fig. 1 An interactive work flow to evaluate operator's plan

\section{Conclusion}

In this paper, we extended the work of [3] to a temporal setting, and designed a set of measurements to evaluate operators' decisions. Our work provides a more expressive and hence powerful tool for decision making. Particularly, our proposed approach models the decision making scenario with predicates, numeric variables and time that are key notions in temporal planning. And, we employed a temporal planning system to compute the optimal plan, which provides a general and scalable way for interactive decision support.

\section{Acknowledgements}

This work was supported by Scientific Research Program of Education Department of Hubei province for Young Talents (Q20151516). 


\section{References}

[1] Bolouri-Yazdeli Y, Haddad O B, Fallah-Mehdipour E, et al. Evaluation of real-time operation rules in reservoir systems operation. Water resources management, 2014, Vol. 28 No. 3, p. 715-729.

[2] Li J, Chen X, Li X, et al. Evaluation of public transportation operation based on data envelopment analysis. Procedia-Social and Behavioral Sciences, 2013, Vol. 96, p. 148-155.

[3] Schubert J, Moradi F, Asadi H, et al. Simulation-based decision support for evaluating operational plans. Operations Research Perspectives, 2015, Vol. 2, p. 36-56.

[4] Vidal V, Geffner H. Branching and pruning: An optimal temporal POCL planner based on constraint programming. Artificial Intelligence, 2006, Vol. 170 No. 3, p. 298-335.

[5] Bylander T. The computational complexity of propositional STRIPS planning. Artificial Intelligence, 1994, Vol. 69 No. 1, p. 165-204.

[6] Fox M, Long D. PDDL2.1: An Extension to PDDL for Expressing Temporal Planning Domains. Journal of Artificial. Intelligence Research.(JAIR), 2003, Vol. 20, p. 61-124.

[7] Micheli A, Do M, Smith D E. Compiling away uncertainty in strong temporal planning with uncontrollable durations. Twenty-fourth international joint conference on artificial intelligence (IJCAI). Buenos Aires, Argentina, July 25-31, 2015, p. 1631-1637.

[8] Cimatti A, Micheli A, Roveri M. Solving strong controllability of temporal problems with uncertainty using SMT. Constraints, 2015, Vol. 20 No. 1, p. 1-29. 